\title{
DÉTECTION ET CARACTÉRISATION SPATIALE DE LA CORROSION LOCALISÉE DES ACIERS AU CARBONE EN MILIEU ANAÉROBIE SULFUROGÈNE
}

\author{
D. Festy' ${ }^{1}$, B. Forest' ${ }^{1}$, M. Keddam ${ }^{2}$, R. Marchal $^{3}$, N. Monfort Moros ${ }^{2.3}$, B. Tribollet $^{2}$ \\ 1. IFREMER, Centre de Brest, Service Matériaux et Structures, Plouzamé \\ 2. UPR 15 du CNRS Laboratoire de "Physigue des Liquides at Electmohimie". Paris \\ 3. Institut Français du Pétrole, Division Chimie et Physico-Chimie Appliquée. \\ Département Microbiologié, Rueil-Malmaison
}

\begin{abstract}
DETECTION AND SPATIAL CHARACTERIZATION OF CARBON STEEL PITTING; CORROSION IN ANAEROBIC SULPHOGENIC MEDIUM

ABSTRACT: The biofilm developing on carbon steel surfaces in anaterobic condition may indure localised corrosion. To be able to better understand this type of biocorrosion, this paper presents a new electrochemical technique. which has been developed in collaboration between IFREMER and the Laboratory for liquid physic and electroche'mistry: Focussed on local aspert of this phenomenon, the described technique enables surface current density mapping to be performed and anodic or cathodic zones to be identified. A double microelectrode probe is placed closed to the steel sample sufface and ponential difference between them is measured. This value is directly connected to ohmic drop within electrolyte and conse'quenty, to local currem. By scanning the substrate surface, local current repartition is visualised and one can detect and characterise localised corrosion antacks. After presenting the tedmique and the calibratom mocedure, a biocorrosion phenomenon induced by scrapping a biofilm al a carbon steel sample surface is analysed by successively drawing localised current maps, included biocide cfficiency assessment.
\end{abstract}

RÉSUMÉ: La présence à la surface des aciers au carbone d'un biofilm développé en milieu anaérobie peut, dans certains cas, induire une altaque de corrosion localisée. Dans le cadre d'une meilleure compréhension de cette biocorrosion, une nouvelle technique électrochimique, développée en collaboration entre l'IFRI:Ml:R el le Laboratoire de Physique des Liquides el d'Electrochimie, est présentée. Destinée à l'étude de l'aspect local de ce type de phénomène. la technique décrite permet d'établir une cartographie de densité de courant sur une surfiace métallique et d'identifier les zones jouant un rôle anodicjue et celles jouant un rôle cathodicjue. Pour cela, une sonde de mesure constituée de deux micro-électrodes est positionnéc alu voisinage de la surface du substrat et la différence de potentiel entre ces deux électrodes est mesurée. permettant d’accéder à la valeur de la chute ohmique dans la solution et, par conséquent, à celle du courant local. Le balayage de la surface étudiée permet de visualiser la répartition des courants locaux et ainsi détecter et caractériser les attiques de corrosion localisée: étendue de lá zone anodique et densité de courant. Suite à la présentation de la technique et de la procédure de mise au point, un phénomène de biocorrosion amorcée mécaniquement sur un coupon métallique est analysé par tracé de cartographies successives, y compris l'évaluattion de l'elficacité d'un biocide.

Contérence présentée au Forum "Biodétérioration des matériaux" organisé les 23 et 24 mai 2002 à Dourdan par la Commission Biodétérioration des Matériaux du Centre français de l'Anticorrosion (Cefracor) avec le parrainage de la Société Française de Microbiologie. 
moins en présence d'un film [12, 13]. La technique de balayage d'électrode à référence (SRET pour Scanning Reference Electrode Technique) a été utilisée pour localiser l'apparition de sites de corrosion localisée dans le cas de corrosion intergranulaire par piqûre et de rupture par corrosion sous contrainte [14-17]. La technique de balayage par électrode vibrante (SVET pour Scanning Vibrating Electrode Technique) est plus [18-20] que la technique SRET dans la détection de courant direct et a été appliquée avec succès dans la détection de défauts dans les revêtements organiques et inorganiques. La Spectroscopie par Impédance Electrochimique a été utilisée pour étudier la dégradation de métaux revêtus exposés à de nombreux environnements [21]. Cependant, l'interprétation des données est généralement diffícile par la complexité des systèmes étudiés et par le fait que les données d'impédance sont relatives à un phénomène généralisé alors que la dégradation se développe souvent sous une forme localisée. La contribution des réactions de corrosion au spectre d'impédance global est difficilement discernable. Plusieurs tentatives pour développer une technique de balayage d'impédance sont rapportées dans la littérature. Une technique utilisait une géométrie de cellule à couche mince. La taille de la sonde était suffisamment large et limitait la résolution spatiale et tout transport de masse prenant place[22].

Une nouvelle méthode de mesure de spectre d'impédance locale a été rapportée par Lillard et al. [23] et récemment étudiée par Bayet et al. [24, 25]. Dans cette méthode, la spectroscopie d'impédance électrochimique locale (LEIS pour Local Electrochemical Impedance Spectroscopy) est déduite des courants alternatifs locaux. La densité de courant alternatif est déterminée à partir des gradients de potentiel au-dessus d'une surface en utilisant un capteur réalisé à cet effet.

Cependant, une meilleure compréhension de l'origine des défauts et des caractéristiques de croissance d'une biocorrosion demande davantage d'information, à un niveau microscopique: aussi bien la répartition des zones électrochimiques que la microstructure du biofilm doivent faire l'objet d'une plus ample recherche. C'est pour cela qu'un capteur électrochimique d'investigation locale en mesure de détecter, localiser et caractériser les défauts engendrés par un processus de biocorrosion permettrait une analyse plus détaillée des facteurs contrôlant l'amorçage et le maintien de la biocorrosion. La technique de cartographie par micro-électrodes développée dans le cadre d'une collaboration entre l'UPR 15 du CNRS et le Service Matériaux et Structures de l'IFREMER participe ainsi à la compréhension de la biocorrosion des aciers au carbone en présence de bactéries sulfatoréductrices, en considérant comme paramètre majeur le courant local au niveau des zones de piqûre de corrosion.

\section{Principe}

La technique de cartographie développée est basée sur la mesure de la différence de potentiel (ddp) entre deux micro-électrodes bloquantes à proximité d'une surface électriquement active. Cette ddp correspond à la chute ohmique au sein de la solution proche de la surface métallique (fig. 1). Le dispositif expérimental développé est constitué d'une sonde de mesure à deux micro-électrodes introduite dans une cellule d'électrochimie montée sur une table $\mathrm{X}-\mathrm{Y}-\mathrm{Z}$.

La sonde de mesure est constituée de deux fils d'argent isolés par un vernis polyuréthanne disposés dans un tube plastique

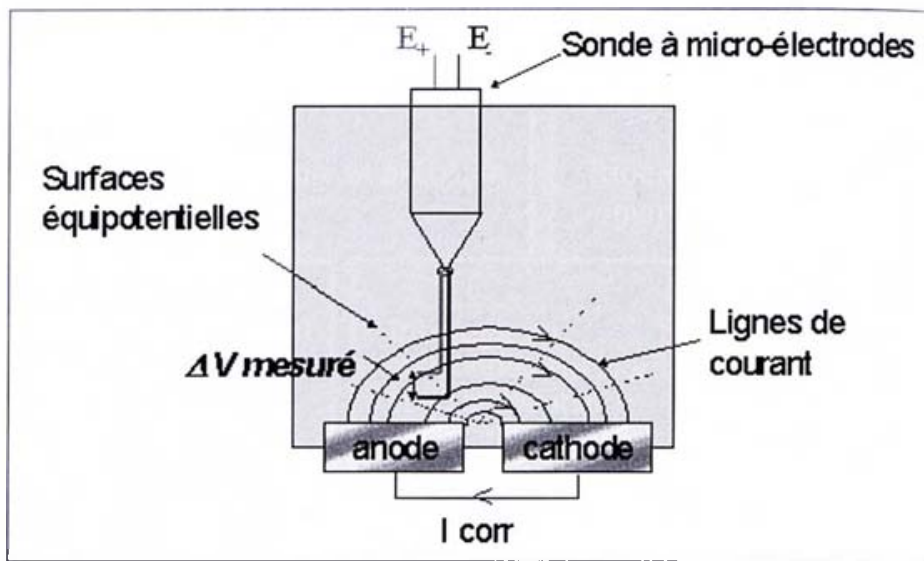

Fig. 1 - Schéma de principe de la mesure de la chute ohmique.

Fig. I-Olmic drop measurement principle.

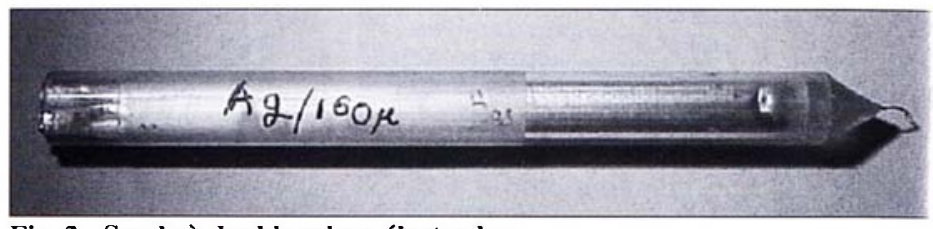

Fig. 2 - Sonde à double micro-électrodes.

Fig. 2 - Double microelectrodes probe.

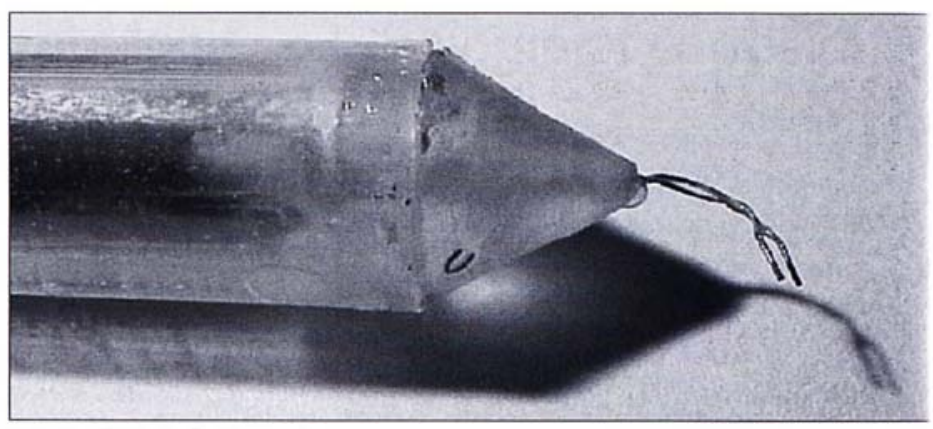

Fig. 3 - Détail de la sonde.

Fig. 3 - Probe detail.

terminé par un cône percé en son centre (figures 2 et 3 ). Le extrémités des fils d'argent, les micro-électrodes, sont position nées parallèlement à l'axe de la sonde et séparées d'une distan ce d'environ $0,4 \mathrm{~mm}$.

Les micro-électrodes sont finalement conditionnées dans unt solution à $3 \%$ de $\mathrm{NaCl}$ afin d'obtenir un film d' $\mathrm{AgCl}$ par pola risation anodique selon la réaction $\mathrm{Ag}+\mathrm{Cl}^{-} \rightarrow \mathrm{AgCl}+\mathrm{e}^{-}$.

Le montage expérimental comprend (fig. 4):

- une cellule électrochimique à double paroi,

- une table X-Y-Z (MM4005, Newport) constituée de trois moteurs pas à pas orientés selon les trois axes orthogonaux,

- un système d'acquisition d'images par caméra vidéo (KPC 550, Hitachi).

La cellule de biocorrosion (fig. 5) est réalisée en Plexiglas L'électrode de travail (coupon cartographié) est positionnée horizontalement au fond de la cellule. Une double paroi permet une régulation thermique de l'enceinte. Une entrée et une sortie sont aménagées pour la circulation d'un liquide dans la cellule ainsi qu'une entrée et une sortie situées sur le couvercle de la cellule pour la circulation d'un gaz dans le ciel de la cellule d'électrochimie. Un joint tubulaire souple est fixé entre le couvercle et la cellule d'électrochimie pour conserver le caractère 


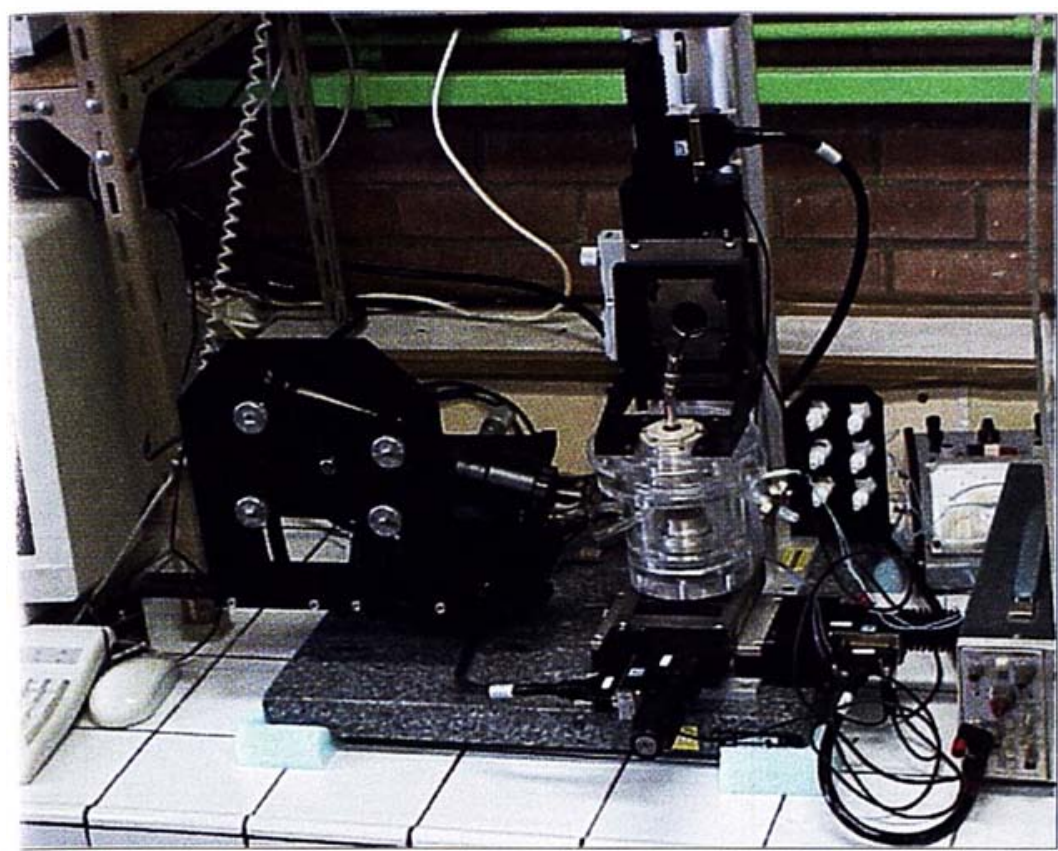

ig. 4 - Montage expérimental pour la réalisation de cartographies de densité de courant. ig. 4 - Current density mapping experimental device.

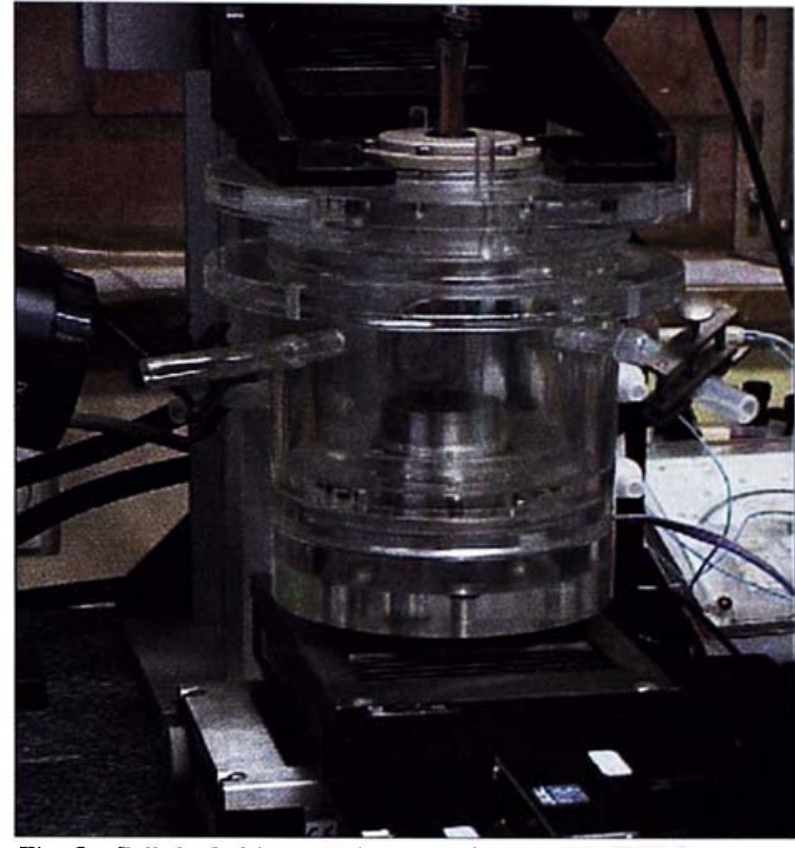

Fig. 5 - Cellule de biocorrosion montée sur table X-Y-Z.

Fig. 5 - Biocorrosion cell set on X-Y-Z table. naérobie à l'ensemble tout en autorisant le déplacement de un par rapport à l'autre.

es mouvements suivants les axes $\mathrm{X}$ et $\mathrm{Y}$ de la table permetent de déplacer la cellule, et donc le coupon, dans le plan horiontal. La troisième motorisation permet d'abaisser ou relever a sonde de mesure, afin d'approcher les micro-électrodes de la urface du coupon de façon reproductible et d'éviter l'arrachenent du biofilm par la sonde lors des déplacements. En effet, le ositionnement initial (distance à la surface du coupon) de la onde est réalisé avec l'aide de la caméra vidéo. Cette position st enregistrée pour réaliser les cartographies car, en présence l'un biofilm, la surface du coupon n'est plus visible. De plus, e déplacement de la sonde de mesure à hauteur constante rovoque l'arrachage du biofilm et peut induire l'amorçage de orrosion localisée [26]. La sonde est donc relevée avant d'être léplacée et repositionnée pour la mesure suivante.

e montage expérimental est complété par une chaîne de nesures composée principalement par un amplificateur difféentiel (AM 502, Tektronix), d'un filtre (VBF8, Kemo) et l'une carte d'acquisition spécialisée équipant un ordinateur. In logiciel adapté permet le pilotage de l'ensemble expérimenal (déplacements de la sonde et mesures).

a densité de courant ponctuelle, $j\left(\mathrm{en} \mathrm{A} / \mathrm{cm}^{2}\right)$, est calculée à artir de la valeur de la chute ohmique (équation 1).

$$
j=\frac{\Delta \Phi}{\rho . d}
$$

4vec: $\Delta \Phi$ la différence de potentiel entre les deux micro-élecrodes (V), $\rho$ la résistivité de l'électrolyte en $(\Omega . \mathrm{cm})$ et $d$ la disance entre les deux micro-électrodes $(\mathrm{cm})$.

\section{Calibration}

La calibration de l'ensemble expérimental décrit est réalisée en disposant dans la cellule électrochimique une électrode disque/ anneau (fig. 6).
- la solution expérimentale est une solution aqueuse de chlorure de sodium $\mathrm{NaCl} 3 \%$ dont la résistivité est de $24 \Omega . \mathrm{cm}$,

- l'électrode de travail est le disque central, d'une surface de $3,14 \mathrm{~mm}^{2}$ (diamètre $=2 \mathrm{~mm}$ ),

- la contre-électrode est l'anneau externe, d'une surface de $326 \mathrm{~mm}^{2}$,

- la surface de balayage de la sonde de mesure à micro-électrode est de $10 \times 10 \mathrm{~mm}$,

- $\Delta \mathrm{X}=0,5 \mathrm{~mm}$,

- $\Delta Y=0,5 \mathrm{~mm}$.

Pour chaque valeur de courant anodique appliqué au disque, une cartographie des courant locaux est réalisée et par intégration directe du profil obtenu (éq. 2) le courant global I (en $\mu \mathrm{A}$ ) détecté est calculé.

$$
I=\int_{X \min }^{X \max } \int_{Y \min }^{Y \max } j(X, Y) d X d Y
$$

où $j(X, Y)$ est la densité de courant au point $(X, Y)$ exprimé en $\mu \mathrm{A} / \mathrm{mm}^{2}, d X$ et $d Y$ en mm.

Fig. 6 -

Electrode dis que / anneau utilisée pour la calibration de la chaîne de mesure.

Fig. 6 -

Diskt ring eiectrode used for calibration procedure.

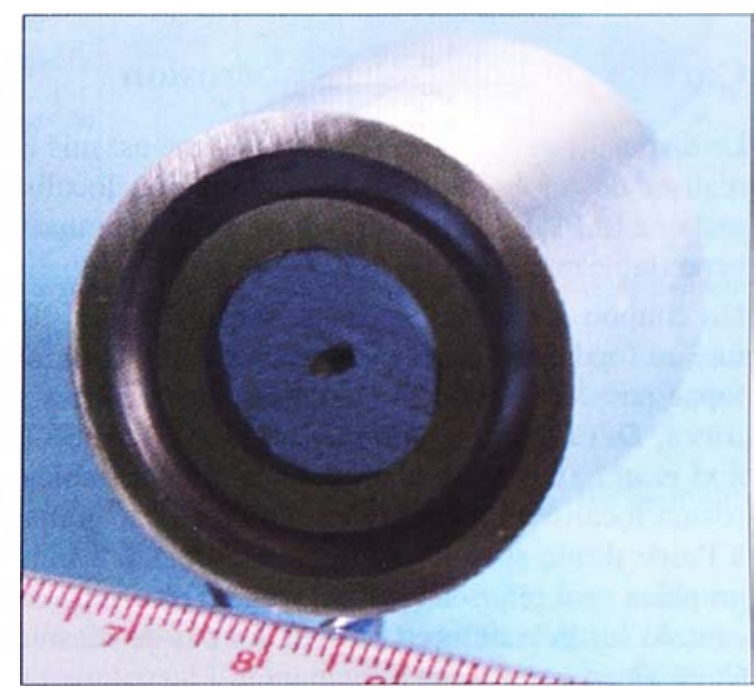




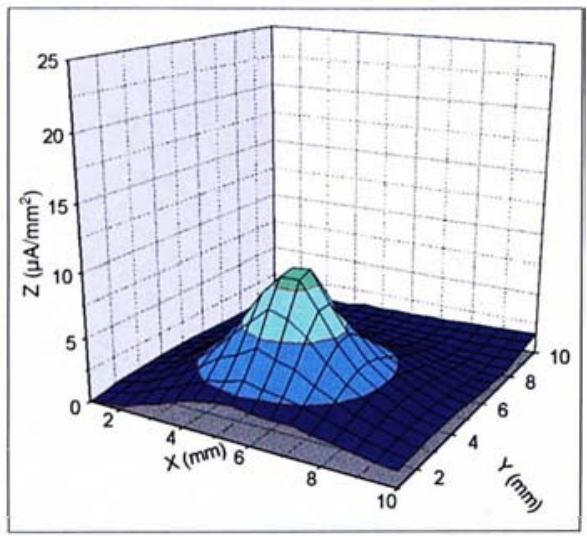

Fig. 7 - Cartographie pour une polarisation de $2 \mu \mathrm{A}$. Fig. 7 -Mapping for $2 \mu$ A polarisation.

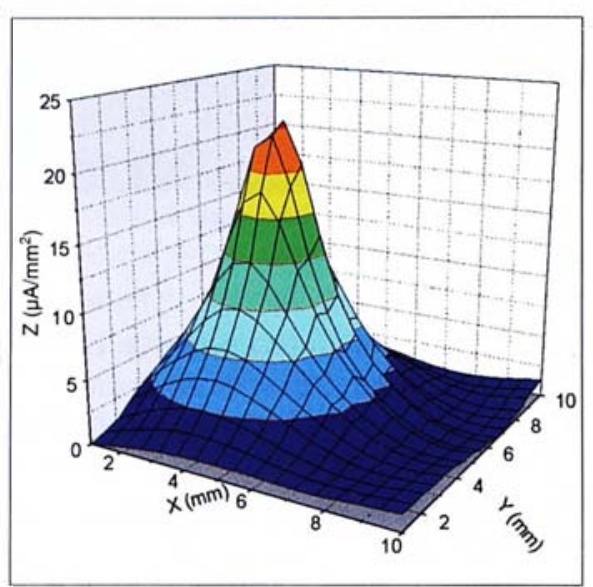

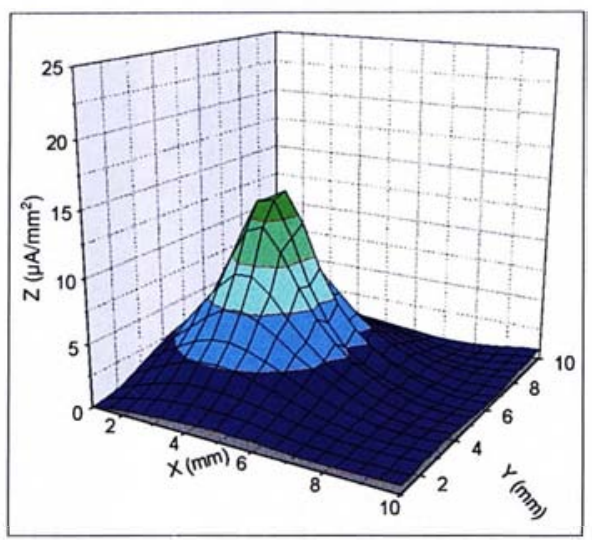

Fig. 8 - Cartographie pour une polarisation de $4 \boldsymbol{\mu A}$.

Fig. 8 - Mapping for $4 \mu A$ polarisation.

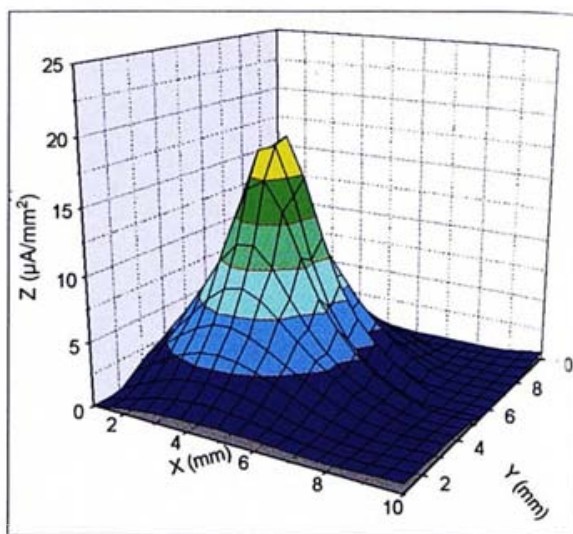

Fig. 9 - Cartographie pour une polarisation de $6 \mu 1$

Fig. 9 - Mapping for $6 \mu$ A polarisation.
Fig. 10 - Cartographie pour une polarisation de $8 \mu \mathrm{A}$.

Fig. 10 - Mapping for $8 \mu$ A polarisation.

Fig. 11 - Courant global mesuré fonction du courant appliqué.

Fig. Il - Overall measured current versus applied current.

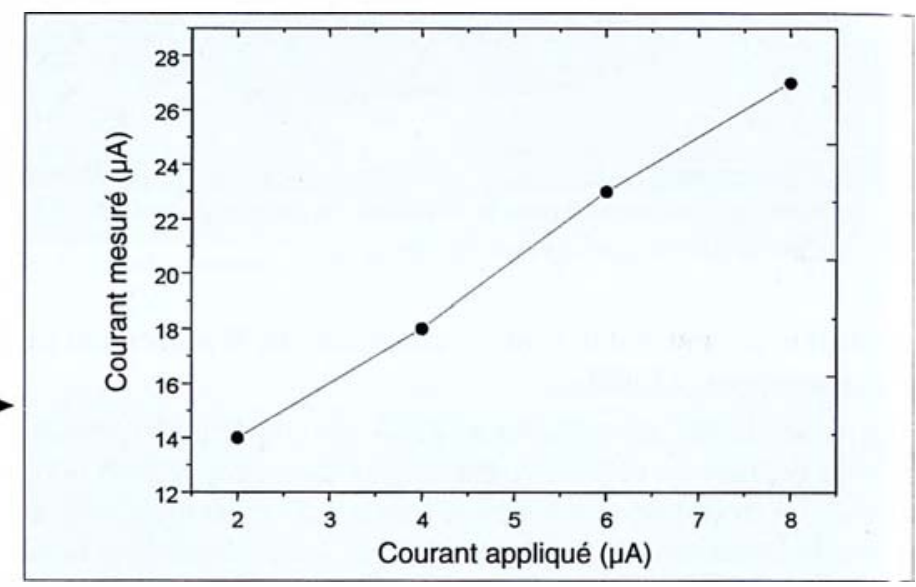

Tableau I - Composition de l'acier API5L Grade 50D.

Table I-APISL steel compositic ।

\begin{tabular}{|c|c|c|c|c|c|c|c|c|c|c|c|c|c|c|}
\hline $\mathbf{C}$ & $\mathbf{C u}$ & $\mathbf{M n}$ & $\mathbf{A l}$ & $\mathbf{S i}$ & $\mathbf{N i}$ & $\mathbf{P}$ & $\mathbf{V a}$ & $\mathbf{S}$ & $\mathbf{S n}$ & $\mathbf{C r}$ & $\mathbf{T i}$ & $\mathbf{M o}$ & $\mathbf{B}$ & $\mathbf{F e}$ \\
\hline 0,063 & 0,264 & 1,480 & 0,052 & 0,481 & 0,362 & 0,0039 & Traces & 0,0006 & 0,0192 & 0,037 & Traces & 0,091 & 0,0004 & Reste \\
\hline
\end{tabular}

Les figures 7 à 10 présentent les cartographies réalisées aux différentes valeurs de courant de polarisation anodique du disque. La corrélation entre l'intensité de courant de polarisation et le courant global déduit de l'intégration des cartographies (fig. 11) est utilisée par la suite comme courbe de calibrage pour quantifier un courant de biocorrosion amorcée artificiellement à la surface d'un coupon.

\section{Cartographies d'une biocorrosion}

Le dispositif expérimental ainsi étalonné est mis en œuvre pour réaliser des cartographies de biocorrosion localisée d'acier au carbone (API5L Garde 50D) dans un milieu anaérobie sulfurogène (tableau I).

Un coupon d'acier au carbone de dimensions $20 \times 30 \mathrm{~cm}$ est fixé au fond de la cellule électrochimique. Un biofilm est développé par décantation d'une culture de bactéries sulfato-réductrices, Desulfovibrio gabonensis DSM 10636 (Tardy-Jacquenod et al., 1996), circulant dans la cellule (tableau II). La corrosion localisée du coupon est amorcée par grattage du biofilm à l'aide d'une spatule (zone grattée de 1 à $3 \mathrm{~mm}^{2}$ ). Les cartographies sont réalisées par balayage d'une zone de $10 \times 10 \mathrm{~mm}$ centrée sur la zone grattée, avec un pas de mesure de $0,25 \mathrm{~mm}$ (X et $\mathrm{Y})$.
Tableau II - Composition en g/L de la solution nutritive des BSR. Table II - Composition of SRB nutritive solution.

\begin{tabular}{|c|c|c|c|}
\hline Sulfate de sodium $\mathrm{Na}_{2} \mathrm{SO}_{4}$ & 2 & Extrait de levure & $0,2^{6}$ \\
\hline $\begin{array}{l}\text { Hydrogénocarbonate } \\
\text { de potassium } \mathrm{KHCO}_{3}\end{array}$ & 0,2 & $\begin{array}{l}\text { Chlorure de calcium } \\
\text { dihydraté } \mathrm{CaCl}_{2}, 2 \mathrm{H}_{2} \mathrm{O}\end{array}$ & 0,1 \\
\hline $\begin{array}{l}\text { Sulfate de magnésium } \\
\text { heptahydraté } \mathrm{MgSO}_{4}, 7 \mathrm{H}_{2} \mathrm{O}\end{array}$ & 4 & $\begin{array}{l}\text { Di-hydrogénophosphate } \\
\text { de potassium } \mathrm{K}_{2} \mathrm{HPO}_{4}\end{array}$ & e \\
\hline Chlorure de sodium $\mathrm{NaCl}$ & 30 & Lactate de sodium & 2,4 \\
\hline $\begin{array}{l}\text { Chlorure d'ammonium } \\
\mathrm{NH}_{4} \mathrm{Cl}\end{array}$ & 1 & $\begin{array}{l}\text { Sulfate de fer hepta- } \\
\text { hydraté } \mathrm{FeSO}_{4}, 7 \mathrm{H}_{2} \mathrm{O}\end{array}$ & $0,15.10^{-}$ \\
\hline $\mathrm{pH}$ & 7,4 & Température & $37,4^{\circ} \mathrm{C}$ \\
\hline
\end{tabular}

La cartographie présentée figure 12 est représentative de $\rfloor$ distribution des densités de courant à la surface du coupo 1 métallique en début d'exposition: le profil obtenu est uniform? et de faible amplitude. Après 5 jours de formation de biofiln, la cartographie obtenue est semblable (fig. 13): la formation $d$ l biofilm n'induit pas d'événement de nature électrochimique.

Juste après le grattage d'une partie du biofilm, la cartographi: réalisée (fig. 14) permet de détecter une zone à fort caractèr ? anodique, exactement à l'endroit de la zone grattée. La densit ś de courant maximale est de $20 \mu \mathrm{A} / \mathrm{mm}^{2}$ et le courant globcl obtenu par intégration des valeurs locales est de $22 \mu \mathrm{A}$. L 1 courbe de calibrage (fig. 11) permet de traiter cette dernièr 


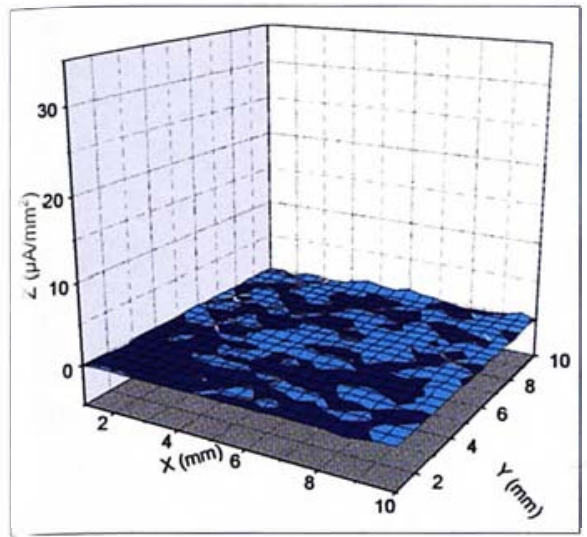

ig. 12 - Cartographie des densités de courant a $r$ un coupon métallique en milieu bactérien : 1 temps initial.

ig. 12 - Current density mapping of a metallic ( 'upon exposed to bacterial media: immersion ine.

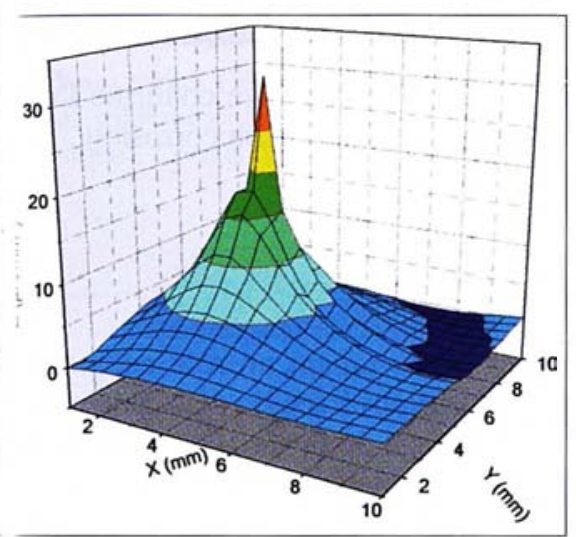

g. 15 - Cartographies des densités de courant ! heures après grattage.

, ig. 15 - Current density mapping of a metallic " 'upon exposed to bacterial media 24 hours after rapping.

aleur en courant circulant sur la surface nodique, soit une valeur de $5,8 \mu \mathrm{A}$. En onsidérant une surface anodique de $\mathrm{mm}^{2}$, la densité de courant de biocorroion est de $1,9 \mu \mathrm{A} / \mathrm{mm}^{2}$, équivalent à une itesse de corrosion de $2,1 \mathrm{~mm} / \mathrm{an}$.

a forte valeur du courant anodique après rattage est en accord avec les valeurs btenues avec une sonde de mesure de la itesse de corrosion localisée des aciers u carbone en milieu anaérobie [26].

'ingt quatre heures après l'amorçage de I biocorrosion (fig. 15) la zone à caractè2 anodique est toujours bien identifiable t s'agrandit, révélant une tendance à la ropagation de la zone corrodée. La aleur maximale du pic de densité de courant anodique est de $31 \mu \mathrm{A} / \mathrm{mm}^{2}$ et le courant global est de $27 \mu \mathrm{A}$. En considérant toujours une surfa'e corrodée de $3 \mathrm{~mm}^{2}$, la vitesse de corrosion peut être évaluée $12,9 \mathrm{~mm} / \mathrm{an}$.

-es cartographies réalisées à 72,96 et 144 h d'exposition figures 16,17 et 18) font apparaître des variations temporelles

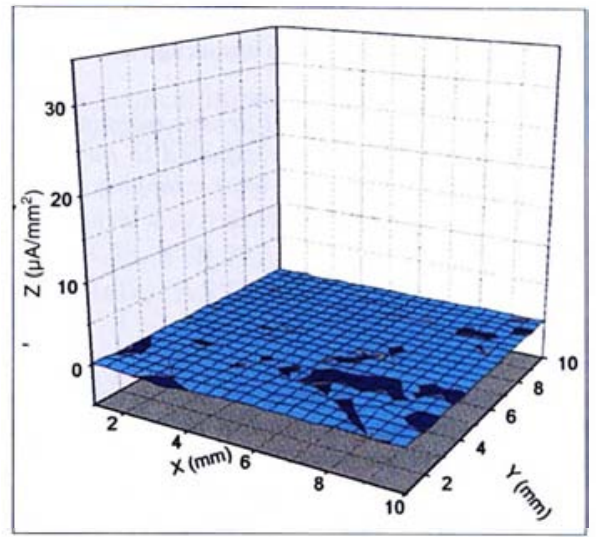

Fig. 13 - Cartographie des densités de courant sur un coupon métallique en milieu bactérien après formation de biofilm.

Fig. 13 - Current density mapping of a metallic coupon exposed to bacterial media: after biofilm developinent.

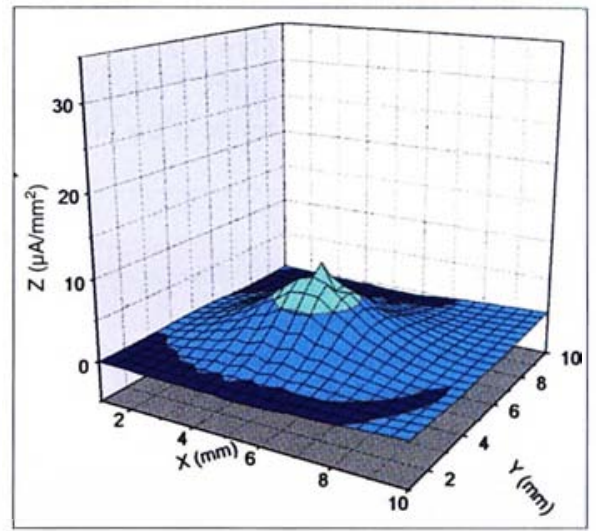

Fig. 16 - Cartographies des densités de courant 72 heures après amorçage mécanique.

Fig. 16 - Currem density mapping of a mo'ullic coupon exposed to bacterial media 72 hours after scrapping.

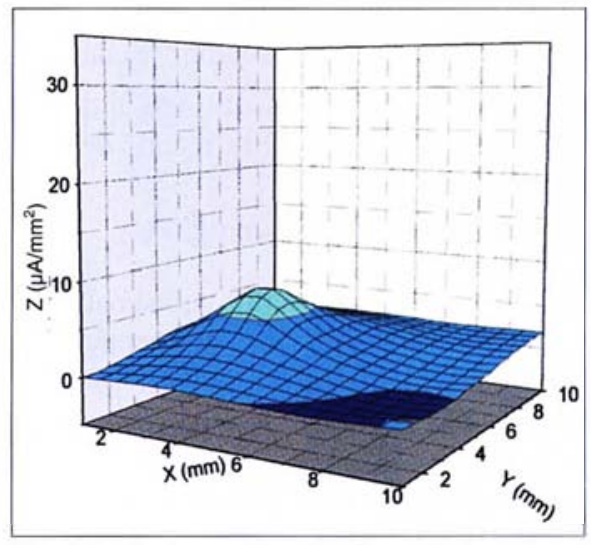

Fig. 18 - Cartographies des densités de courant 144 heures après amorçage mécanique.

Fig. 18 - Current density mapping of a metallic coupon exposed to bacterial media 144 hours after scrapping.

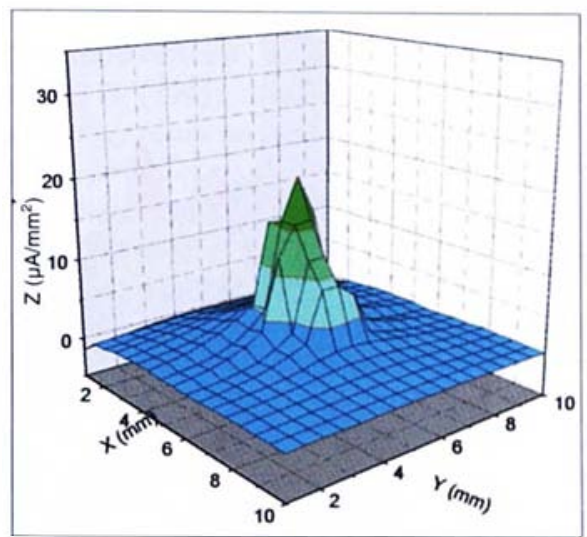

Fig. 14 - Cartographies des densités de courant immédiatement après grattage.

Fig. 14 - Current density mapping of a me'allic coupon exposed to bacterial media after icraplying.

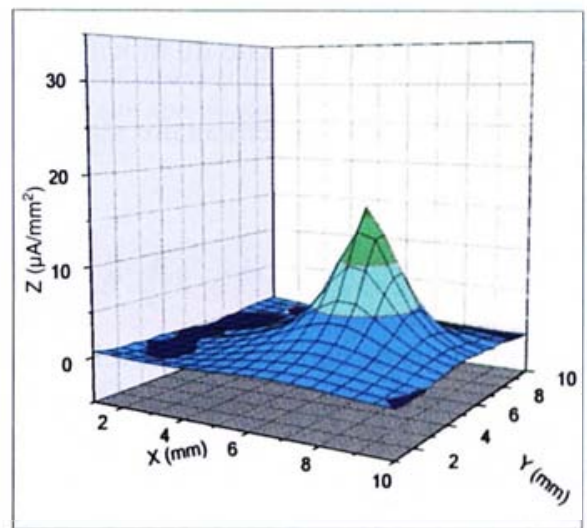

Fig. 17 - Cartographies des densités de courant 96 heures après amorģage mécanique.

Fig. 17 - Currem densiuy mapping of a meallic coupon exposed to bacterial modia go hours afier scropping.

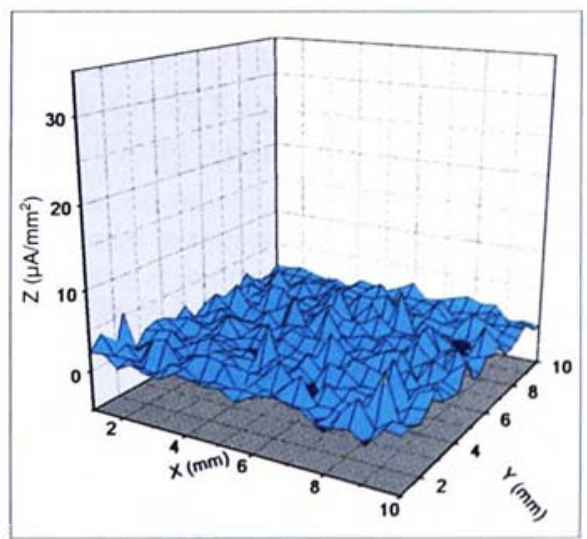

Fig. 19 - Cartographies des densités de courant $\mathbf{2 4} \mathrm{h}$ après ajout d'eau de Javel.

Fig. 19 - Current density mapping of a metallic coupon exposed to bacterial media after biocide ireamineat.

de la vitesse de corrosion localisée, en accord avec les résultats obtenus par ailleurs [26].

Cent quarante quatre heures après le grattage, une solution d'eau de Javel à 20 ppm est injectée dans la cellule d'électrochimie tout en maintenant l'alimentation en solution bactérienne 


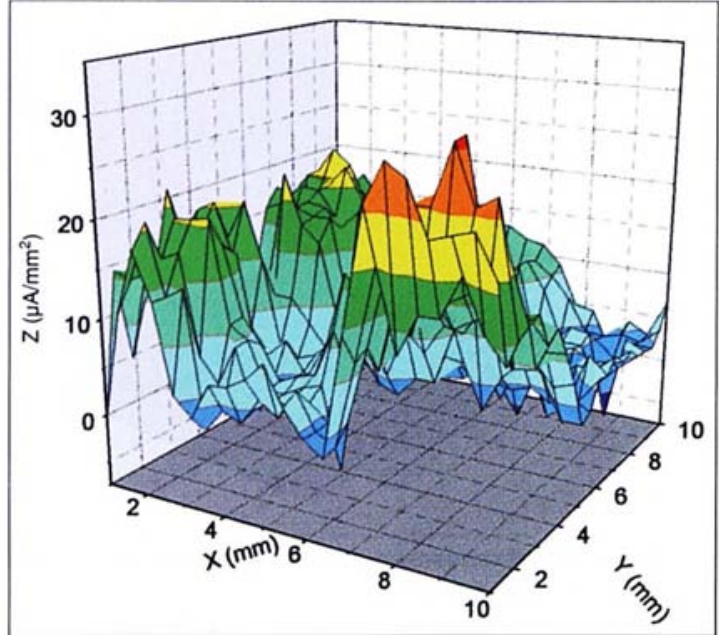

Fig. 20 - Densités de courant 144 heures après amorçage mécanique de biocorrosion.

Fig. 20 - Current density mapping of a metallic coupon exposed to bacterial media 144 hours after scrapping.

\section{Fig. 21 - Piqûres de biocorrosion à la surface du coupon métallique. Le carré en pointillé de $10 \times 10 \mathrm{~mm}$ cor- respond à la zone cartographié de la figure 17. \\ Fig. 21 - Pitting corrosion of metallic coupon. $10 \times 10 \mathrm{~mm}$ dot square corres- pond to mapped zone (fig. I7).}

afin de soumettre le biofilm à un traitement choc. La cartographie (fig. 19) effectuée $24 \mathrm{~h}$ après ce traitement est identique aux cartographies réalisées pendant la formation de biofilm, montrant ainsi l'efficacité du biocide sur l'inhibition de ce type de biocorrosion.

Une expérience complémentaire réalisée afin de comparer profil

\section{Conclusions}

La technique de cartographie développé est basée sur la mesure de la différence de potentiel (ddp) entre deux micro-électrodes bloquantes à proximité d'une surface électriquement active. Cette ddp correspond à la chute ohmique au sein de la solution proche de la surface métallique (fig. 1). Le dispositif expérimental développé est constitué d'une sonde de mesure à deux micro-électrodes introduites dans une cellule d'électrochimie montée sur une table $\mathrm{X}-\mathrm{Y}-\mathrm{Z}$.

Une meilleure compréhension des phénomènes de corrosion localisée comme ceux dans lesquels sont impliqués les bactéries sulfurogènes nécessite la mise en œuvre de techniques d'investigation à même les surfaces corrodées. La technique électrochimique permet d'établir une cartographie de densité de courant sur une surface métallique et d'identifier les zones de cartographie (fig. 20) et observation visuelle (fig. 21) $144 \mathrm{~h}$ après l'amorçage de la corrosion par grattage confirme l'exis tence d'un phénomène de propagation de la biocorrosion.

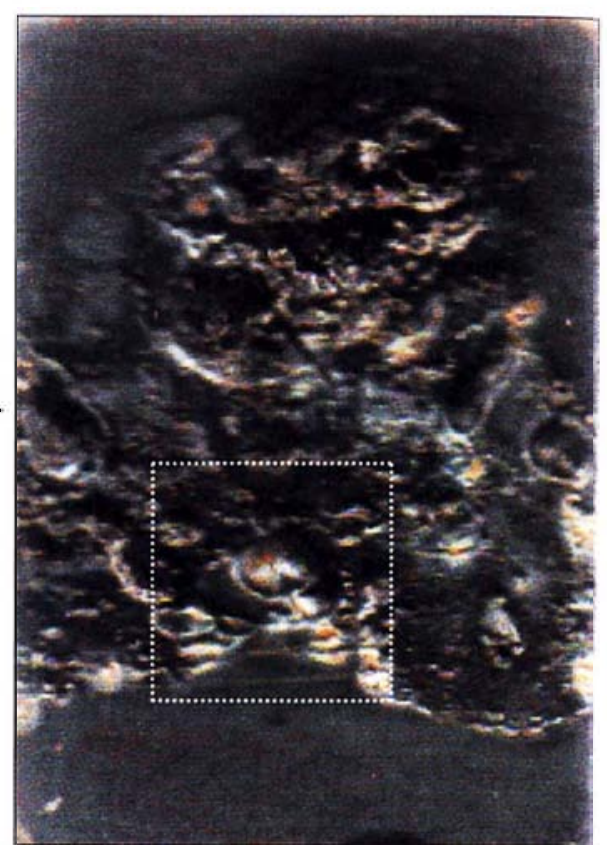

jouant un rôle anodique et celles jouant un rôle cathodique Pour cela, une sonde de mesure constituée de deux micro-élec trodes est positionnée au voisinage de la surface du substrat e la différence de potentiel entre ces deux électrodes est mesurée permettant d'accéder à la valeur de la chute ohmique dans li solution et par conséquent, à celle du courant local. Après mist au point et calibrage de la technique décrite, l'application à uı cas réel de corrosion localisée en présence de bactéries sulfato réductrices a permis de visualiser la répartition des courant: locaux et ainsi de délimiter en fonction du temps (tracés dt cartographies successives) l'étendue de la zone anodique e évaluer les densités de courant.

Cette technique permet également d'évaluer l'impact et don l'efficacité de biocides et d'inhibiteurs de corrosion.

\section{RÉFÉRENCES}

[1] C. Tardy-Jacquenod, P. Caumette, R. Matheron, C. Lanau, O. ARnAUd and M. Magot, Canadian Journal of Microbiology, Vol. 42, pp. 259-266, 1996.

[2] J.L. Crolet, Corrosion 95, Paper $\mathrm{N}^{\circ} 188$ (Houston TX, NACE, 1995).

[3] "Factors influencing accelerated corrosion of cargo oil tanks", Oil Companies International Marine Forum, Sept. 1997.

[4] J. Guezennec, M.W. Mittleman, J. Bullen, D.C. White and J.L. Crolet, Proceedings of UK Corrosion 92, London, UK, Institute of Corrosion.

[5] X. Campaignolle, J.S. Luo, J. Bullen, D.C. White, J. GuezenNEC and J.L. Crolet, Corrosion 90, paper $\mathrm{N}^{\circ} 103$ (Houston TX NACE, 1993).

[6] X. Campaignolle, PhD thesis, Institut National Polytechnique de Toulouse, November 1996.

[7] X. Campaignolle and J.L. Crolet (97), Corrosion, Vol. 53, $\mathrm{N}^{\circ} 6$, pp 440-447.
[8] Annergren I., Thierry D., and Zou F. (1997), 'Localized elec trochemical impedance spectroscopy for studying pitting corro sion on stainless steels', J. of Electrochem. Soc., 144 (4), 1208.

[9] IsAACS H.S. (1987), 'The use of scanning vibrating electrode technique for detecting defects in ion vapor-deposited aluminum on steel', Corrosion, 43, 594.

[10] Bayet E., Huet F., Keddam M., Ogle K., and Takenouti H (1997), 'A novel way of measuring local electrochemical impedance using a single vibrating probe', J. Electrochem. Soc., 144 (4), L87.

[11] Zhao J.G., Frankel G., and MCCreery R.L. (1998), 'Cortosior protection of untreated AA-2024-T3 in chloride solution by a chromate conversion coating monitored with Raman spectroscopy', J. Electrochem. Soc., 145, 2258.

[12] Whitham T.S. (1995), 'Challenges to the prediction and monitoring of microbially influenced corrosion in the oil industry' Microbial Corrosion, Proceedings of the $3^{\text {rd }}$ International EFC 
Workshop, Tiller A.K. and C.A.C. Sequeira, Editors, EFC Publications $\mathrm{N}^{\circ} 15$, The Institute of Materials.

[13] Zou F. and THIERry D. (1997), 'Localized electrochemical impedance spectroscopy for studying the degradation of organic coatings', Electrochem. Acta, 42, 3293.

[14] IsAACS H.S. and VYAS B. (1981), in Electrochemical Corrosion Testing, Mansfeld F. and Bertocci U., Editors, p.3-33, STP 727, American Society for Testing and Materials, ASTM, Philadelphia, USA.

[15] PA ISAACS H.S (1988), 'Initiation of stress corrosion cracking of sensitized type 304 stainless steel in dilute thiosulfate solution', J. Electrochem. Soc., 135, 2180.

[16] Bates S.J., Gosden S.R.and Sargeant D.A. (1989), 'Design and development of scanning reference electrode technique for investigation of pitting corrosion in EV448 gas turbine disc steel', Mater. Sci. Technol., 5, 356.

[17] Rosenfeld I.L. and Danilov I.S. (1967), 'Electrochemical aspects of pitting corrosion', Corrosion Science, 7, 129.

[18] IsAACS H.S. (1987), 'The use of scanning vibrating electrode technique for detecting defects in ion vapor-deposited aluminum on steel', Corrosion, 43, 594.

[19] ISAACS H.S. (1988), 'The measurement of the galvanic corrosion of soldered copper using the scanning vibrating electrode technique', Corros. Sci., 28 (6), 547.
[20] JAFFE L.F., NuTICELLI R. (1974), 'An ultrasensitive vibrating probe for measuring steady electrical currents', J. Cell. Bio., 63, 614.

[21] Amirudin A. and Thierry D. (1995), 'Comparative evaluation of alkyd, bituminous, and epoxy paints on steel in chloride media by impedance spectroscopy', Brit. Corros. J., vol. 26 (3), 195

[22] IssaCs H.S. and Kendig M.W. (1980), 'Determination of surface inhomogeneities using a scanning probe impedance technique', Corrosion, 36, 269.

[23] Lillard R.S, Moran P.J. and Isaacs H.S. (1992), 'A novel method for generating quantitative local electrochemical impedance spectroscopy', J. Electrochem. Soc., 139 (4), 1007.

[24] Bayet E., Huet F., Keddam M., Ogle K and Takenouti H. (1998), 'Adaptation of the Scanning Vibrating Electrode Technique to ac mode : Local Electrochemical Impedance Measurement', Electrochemical Methods in Corrosion Research VI, Bonora P.L. and Deflorian F., Editors, Materials Science Forum, Trans Tech Publications, Trento, vol. 289-292, 57.

[25] Bayet E., Huet F., Keddam M., Ogle K. and Takenouti H (1999), 'Local electrochemical impedance measurement : scanning vibrating electrode technique in $\mathrm{AC}$ mode', Electrochim. Acta, AA, 4117

[26] MONFORT Moros N. (2001) Corrosion localisée des aciers au carbone induite par des bactéries sulfato-réductrices. Développement d'un capteur spécifique. $\mathrm{PhD}$ thesis, Université de Paris VI, November 2001.

\section{Par qui furent découverts les corps simples de la cbimie?}

\section{LES ÉlÉments Chimiques et les hommes}

Le récit d'une grande aventure

Par ce livre Jean Talbot ravive les péripéties de la découverte des corps simples, de l'antiquité à nos jours. Le lecteur, familier ou non de la chimie, envisage celle-ci sous un nouveau jour et se trouve ainsi associé à l'une des épopées cruciales de l'histoire de l'humanité. Au rythme de l'enchaînement des découvertes, se perçoit la volonté des savants d'éclairer de mieux en mieux les zones d'ombre du savoir.

\section{Les éléments}

Cet ouvrage nous livre tout d'abord des clefs de la classification de Mendéléev, qui sert de guide pour présenter tous les éléments chimiques. Pour chaque élément est alors précisé l'origine de son nom, à quelle date, par qui et comment il fut découvert.

\section{Les hommes}

Puis, au travers de brèves biographies, Jean Talbot relate certains traits de la vie des hommes ayant contribué à accroître le savoir dans le domaine de la chimie. L'auteur dépeint leur caractère, leur carrière, leurs écrits, leur rayonnement dans d'autres domaines, l'opinion des confrères tant sur eux-mêmes que sur

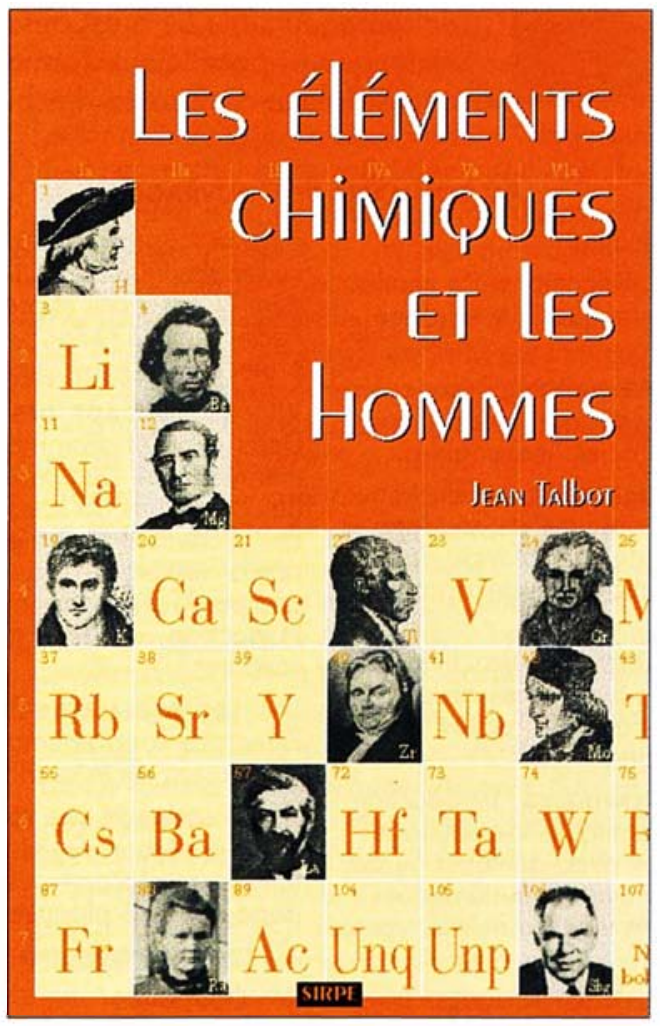

leurs recherches, et souligne les circonstances des découvertes. Les anecdotes relatées et les portraits de la plupart des protagonistes ouvrent à une meilleure connaissance de ces pionniers et de leurs travaux

Les techniques, l'enseignement

Enfin, pour situer le cadre de travail des chimistes des siècles passés, sont abordées l'évolution de certaines techniques de laboratoire qui, dès leur mise en auvre, contribuèrent à do grandes avancées de la recherche, et la genèse et le développement de l'enseignement de la chimie et de la pharmacie en France.

\section{L'auteur}

Jean Talbot $(t)$, professeur honoraire à l'Université Pierre et Marie Curie et ancien directeur de l'Ecole Nationale Supérieure de Chimie de Paris, témoigne d'une sensibilité et d'une ouverture d'esprit qui transparaissent dans ce livre original, procurant à tous un vif plaisir de lecture, qui passionnera le professionnel, l'enseignant, l'étudiant et tous ceux intéressés à connaître des hommes et leurs idées ayant contribué à l'évolution du monde moderne.

1 volume $15,5 \times 24 \mathrm{~cm}$ relié, sous jaquette; 240 pages, 95 illustrations. Prix franco: $185 \mathrm{~F}$ (TVA 5,5\% et port inclus) SIRPE éditeur, 76 rue de Rivoli, 75004 Paris - Tél. (1) 42785220 - Fax (1) 42744048 


\section{SOUDAGE DES ACIERS INOXYDABLES: LES CONNAISSANCES INDISPENSABLES}

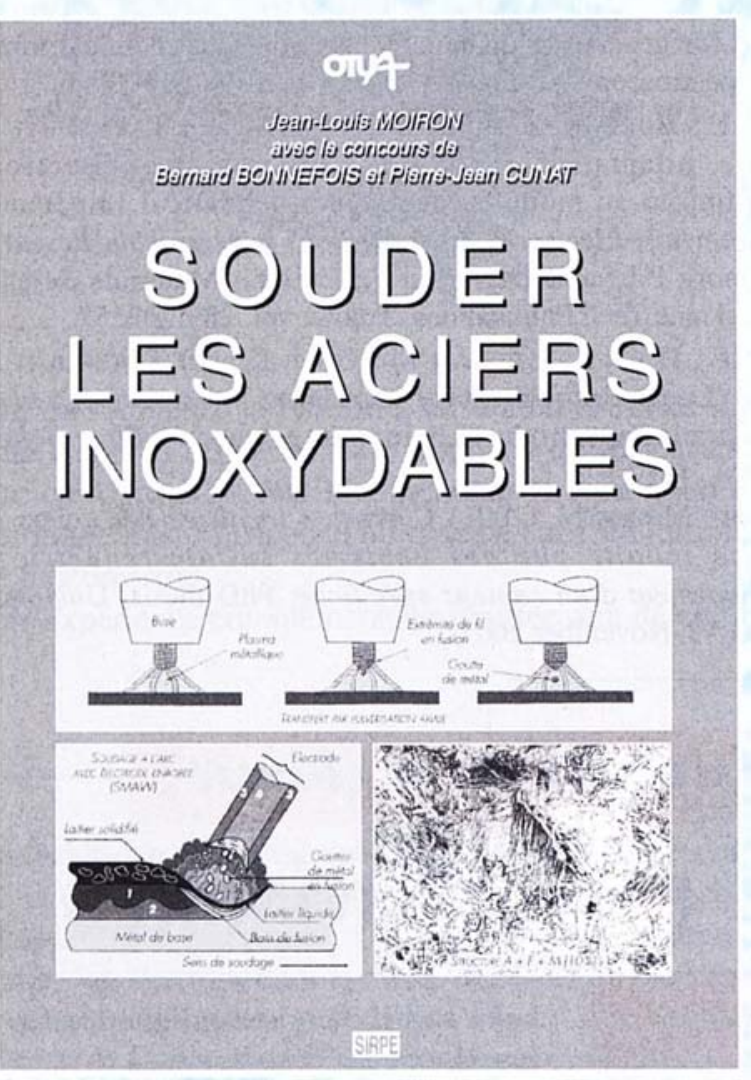

es opérations de soudage des aciers inoxydables requièrent des précautions spécifiques appropriées à leur structure métallurgique, selon qu'il s'agit de mettre en œuvre:

- les aciers inoxydables ferritiques;

- les aciers inoxydables martensitiques;

- les aciers inoxydables austénitiques;

- les aciers inoxydables super-austénitiques;

- ou les aciers inoxydables austéno-ferritiques.

Complément indispensable du manuel "Travailler les aciers inoxydables" publié par SIRPE en 1998, ce livre technique présente d'une manière pragmatique les données de la métallurgie du soudage indispensables pour obtenir des assemblages sains présentant les caractéristiques désirées. II prend en compte les plus récents progrès de la sidérurgie et des savoir-faire.

Elaboré par Jean-Louis MOIRON, Chef du Service Inox du Centre Matériaux "Techniques de soudage" d'Usinor Recherche et Développement, avec le concours de Bernard BONNEFOIs/Centre de Recherche des matériaux du Creusot d'Usinor Recherche ef Développement) et de Pierre-Jean CUNAT (Directeur technique d'Euro-Inox), ce livre constitue la synthèse de l'ancien guide édité par l'ATS et l'OTUA et de celui réalisé par Ugine.

Utile aux entreprises artisanales et aux PMI comme aux grands entrepreneurs, ce livre est aussi un outil précieux pour l'enseignements technique et pour "l'auto-formation" permanente des techniciens. Aussi attil sa place dans toutes les bibliothèques techniques.

\section{Au SOMmaIRe de L'OUVRAGe}

I - Les ACIERS INOXYDABLES : Définition - Classification

II - Rappels sur la métallurgie du soudage : Définitions - Le soudage par fusion - Conséquences des cycles thermiques de soudage Quelques données quantitatives.

III - Les PRINCIPAuX Procédés de soudage par fusion: Description générale - Description des principaux procédés de soudage par fusion. Préparations de joints utilisées pour les aciers inoxydables.

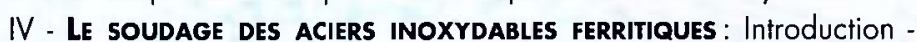
Conséquences métallurgiques des cycles thermiques de soudage Choix des conditions de soudage - Soudage des aciers inoxydables ferritiques avec d'autres types d'aciers - Nettoyage, décapage et passivation.

V - LE SOUdAGE des ACIERS INOXYDABles martensitiques: Introduction - Conséquences métallurgiques des cycles thermiques de soudage - Choix des conditions de soudage - Soudage des aciers inoxydables martensitiques avec d'autres types d'aciers.

VI - LE SOUdAGE deS ACIERS INOXYDABLES AUSTÉnitiqueS : Introduction - Conséquences métallurgiques des cycles thermiques de soudage Soudage des aciers inoxydables austénitiques avec quelques \% de ferrite - Soudage des aciers inoxydables "purement" austénitiques Soudage des aciers inoxydables austénitiques avec d'autres types d'aciers - Nettoyage, décapage et passivation.

VIII - LE SOUDAGE DES ACIERS INOXYDABLES SUPER-AUSTÉNITIQUES : Intro- duction - Principaux matériaux - Conséquences métallurgiques des cycles thermiques - Sur-alliage et résistance à la corrosion de la soudure - Compacité et propriétés mécaniques des soudures (exemple de l'acier UNS S3 1266) - Procédés de soudage utilisables - Les gaz de protection - Nettoyage, décapage et passivation.

VII - LE SOUdAGE dES ACIERS INOXYdABLES AUSTÉNO-FERRItIQUES Description el désignation des aciers austéno-ferritiques - Traitement thermique de qualité et traitement thermique post-soudage - Choix des conditions de soudage - Procédés de soudage utilisables - Choix des produits d'apport et composition du métal déposé - Soudage des aciers austéno-ferritiques avec d'autres aciers - Nettoyage et décapage; passivation des soudures - Détermination de la teneur en ferrite Inspection, contrôle non destructif et essais de qualification des soudures.

IX - Les assemblages hétérogènes : Définition - Introduction Principes fondamentaux - Exemples de soudages hétérogènes Conclusion sur le soudage hétérogène.

$X$ - Le soudage des tôles plaquées : Introduction - Rappel des principes généraux de mise en œuvre - Cas particulier du soudage des tôles avec placage austéno-ferritique - Traitement thermique post-soudage des tôles plaquées.

\section{XI - Le PARACHEVEMENT deS SOUdURES}

XII - GlossaIRE

\section{"Souder les aciers inoxydables"}

1 vol. broché $15 \times 21 \mathrm{~cm}, 144$ pages, 69 illustrations, 42 tableaux, ISBN 2-906643-21-1; prix franco: 45,12€ TTC (41,92€ + port 3,20 €). SIRPE éditeur, 76 rue de Rivoli, 75004 Paris. Tél. 0142785220 - Fax 0142744048

E-mail sirpe@noos.fr - Internet www.sirpe.com 